\title{
Use of Traditional and Novel Methods to Evaluate the Influence of an EVA Glove on Hand Performance
}

\author{
Elizabeth A. Benson ${ }^{1}$ and Scott A. England ${ }^{2}$ \\ MEI Technologies, Houston, TX, 77058 \\ Miranda Mesloh ${ }^{3}$ and Shelby Thompson, $\mathrm{PhD} .^{4}$ \\ Lockheed Martin, Houston, TX, 77058 \\ and \\ Sudhakar Rajulu ${ }^{5}$ \\ NASA, Houston, TX, 77058
}

\begin{abstract}
The gloved hand is one of an astronaut's primary means of interacting with the environment, and any restrictions imposed by the glove can strongly affect performance during extravehicular activity (EVA). Glove restrictions have been the subject of study for decades, yet previous studies have generally been unsuccessful in quantifying glove mobility and tactility. Past studies have tended to focus on the dexterity, strength, and functional performance of the gloved hand; this provides only a circumspect analysis of the impact of each type of restriction on the glove's overall capability. The aim of this study was to develop novel capabilities to provide metrics for mobility and tactility that can be used to assess the performance of a glove in a way that could enable designers and engineers to improve their current designs. A series of evaluations were performed to compare unpressurized and pressurized $(4.3 \mathrm{psi})$ gloved conditions with the ungloved condition. A second series of evaluations were performed with the Thermal Micrometeoroid Garment (TMG) removed. This series of tests provided interesting insight into how much of an effect the TMG has on gloved mobility - in some cases, the presence of the TMG restricted glove mobility as much as pressurization did. Previous hypotheses had assumed that the TMG would have a much lower impact on mobility, but these results suggest that an improvement in the design of the TMG could have a significant impact on glove performance. Tactility testing illustrated the effect of glove pressurization, provided insight into the design of hardware that interfaces with the glove, and highlighted areas of concern. The metrics developed in this study served to benchmark the Phase VI EVA glove and to develop requirements for the next-generation glove for the Constellation program.
\end{abstract}

\section{Nomenclature}

$C C D=$ Cursor Control Device

$E M U=$ Extravehicular Mobility Unit

EVA $=$ Extravehicular Activity

$P G T \quad=$ Pistol Grip Tool

$T M G \quad=$ Thermal Micrometeoroid Garment

\footnotetext{
${ }^{1}$ Human Factors Design Engineer, ABF/SF3, 2101 NASA Parkway, Houston TX 77058

${ }^{2}$ Human Factors Design Engineer, ABF/SF3, 2101 NASA Parkway, Houston TX 77058

${ }^{3}$ Human Factors Design Engineer, ABF/SF3, 2101 NASA Parkway, Houston TX 77058

${ }^{4}$ (Shelby's job title), UTAF/SF3, 2101 NASA Parkway, Houston TX 77058

${ }^{5}$ NASA Lab Manager, ABF/SF3, 2101 NASA Parkway, Houston TX 77058
} 


\section{Introduction}

$\mathrm{T}$ He difficulties associated with building a safe and efficient spacesuit are perhaps at their pinnacle in the design of a spacesuit glove. In a way, the glove represents a microcosm of the entire suit, where all of the complex mobility joints in the pressure garment must be miniaturized for application to the hand. However, the glove is also one of the most crucial areas of the suit because it is the suited human's primary means of interacting with their environment. The glove has been a source of great chagrin to astronauts in the past, and was one of the areas of the suit that the original Apollo astronauts pointed out as needing redesign [1]. There have been many changes and enhancements in EVA glove design since the Apollo era, but as this study determined, there is still room for improvement.

\section{A. The EVA Glove}

In building an Extravehicular Activity (EVA) glove, engineers strive to preserve as much as possible of the human hand's natural dexterity and tactility, but must still protect the hand from the harsh environment of space. The pressurization of the suit leads to inflexibility in the glove, which is exacerbated by the necessary addition of a Thermal Micrometeoroid Garment (TMG). The TMG is a removable outer layer meant to protect the astronaut when they are outside the vehicle, and is composed of multiple layers of material, which can highly impact the mobility of the glove.

The operating pressure and the TMG can also have a noticeable effect on the gloved strength of the human hand, since the wearer is fighting against the pressure of the suit and the friction between TMG layers. The glove also leads to issues with tactility, or the discrimination of features using touch. Lack of tactility can potentially be an issue because the astronaut's field of view is generally restricted in an EVA suit. The effects of reduced strength, mobility and tactility are reflected by the increased time that is required to perform some activities while wearing spacesuit gloves.

\section{B. Benchmarking the EVA Glove}

Since the beginning of the space program, designers have been looking for ways to quantify the performance of spacesuit gloves, in order to evaluate different glove designs and to determine where improvements can be made $[2,3,4,5,6,7]$. The purpose of the current study was to

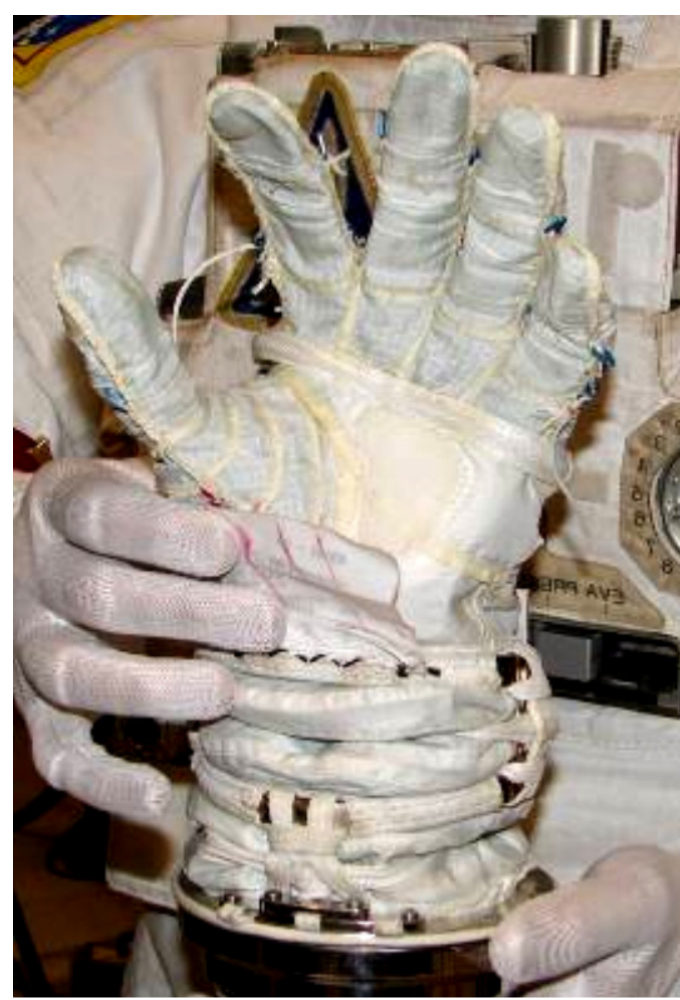

Figure 1: The Phase VI Glove with TMG Removed provide requirements for a new glove, by benchmarking the current EVA glove, the Phase VI. Testing was completed by personnel from the Habitability and Human Factors Branch at NASA's Johnson Space Center, with funding provided by the Constellation EVA Project Office, and with significant support from the Crew and Thermal Systems Division. The intent was to ensure that the next generation glove would perform as good as or better than the current glove. To this end, the authors chose to concentrate on the glove's mobility, its affect on applied strength and tactility, and its impact on performance in completing a set of representative EVA tasks. At the same time, subjective measures of discomfort, glove fit and performance were collected.

\section{Study Definition}

In order to provide useful and accurate requirements for the glove designers, test methods were chosen based on their repeatability, reliability, and ease of application. A series of tests were proposed based on past studies, and some new, innovative methods were also developed. This long list of tests was then down-selected to shorten the length of the overall test.

\section{Philosophy of Glove Testing}

There was some initial discussion regarding the philosophy of this glove testing, and how it would benefit the suit contractor and the engineering and human factors stakeholders. Although many glove tests had been conducted 
in the past, there was limited data available on the Phase VI, which was considered the state of the art in glove design, and currently in use on orbit. The decision was made to benchmark these gloves, and to use their capabilities as the baseline for future glove technologies. This benchmarking could provide requirements for the glove designers to work to, and allow the engineering group to ensure that they were getting a glove that was as good, or better, than current technology. This data could also be provided to tool and vehicle designers, to allow them to develop interfaces that the glove could successfully interact with.

Initially, preference was given to selection of methods that could be readily reproduced by the contractor, so that they could check their prototype glove performance against the standard. However, it was determined that some metrics (such as gloved mobility) could be best evaluated using technology that would not be readily available to an offsite contractor. Other simpler metrics such as strength, however, could easily be checked by the contractor as they developed a new design.

\section{Evaluation of Past Glove Studies}

Before a detailed plan for the current glove study was laid out, extensive research was done to select testing methods. The reviewed literature included previous EVA glove studies, papers on both gloved and ungloved strength measurement, papers on established dexterity tests, and evaluations of novel methods for collecting hand mobility. One particularly pertinent and useful source of information was a (date) study conducted by HinmanSweeney et al [2], in which they described a wide range of tests that had been evaluated for inclusion in an exhaustive EVA glove study. These authors considered multiple testing methods for each gloved attribute including dexterity, tactility, mobility, strength and functional performance. Other applicable glove studies included multiple studies by Rajulu, Bishu and Klute [3,4,5], and studies by Akin et al in 2001 [6,7].

\section{E. Selection of the EMU as a Testbed}

Two options were considered for bringing the spacesuit glove up to its operating pressure. One option was to have the subjects don a spacesuit normally and to provide the test articles at a comfortable operating height for them to interact with. The other option was to use a differential pressure glove box. When a glove box is used, the subject dons spacesuit arms only, and extends their hands into a chamber whose pressure is dropped below ambient. The advantage of this method is that the subject does not to be trained, and never experiences a change in pressure (which they would see during pressurization of a space suit). For this reason, subjects without spacesuit experience and with less stringent medical requirements could be test subjects.

However, the advantage of using a spacesuit is the higher fidelity. When using a glovebox, the subject can vary the amount that they push into the gloves by backing up or pushing forward. In a suit, the pressure applied by the gloves to the subject's fingertips is a function of the suit fit (e.g. arm length), and will vary based on their posture. This would mean, however, that only subjects with an adequate suit fit and appropriate medical clearance could participate

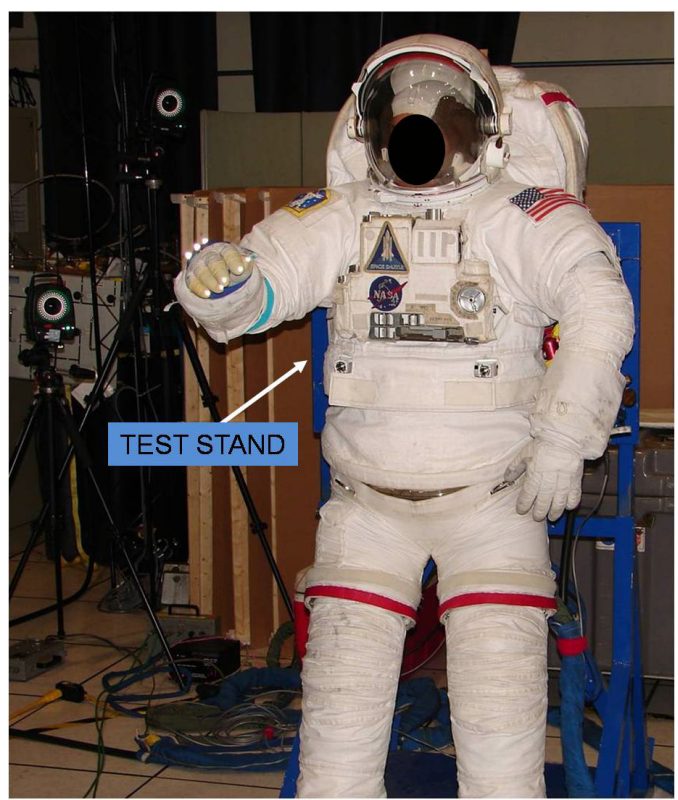

Figure 2. Shuttle EMU in Test Stand. in the study.

For this study, the option of testing in a full space suit was selected to ensure a more realistic posture and fingertip loading. The selected space suit was the current space shuttle Extravehicular Mobility Unit (EMU). Subjects completed a series of tests while the weight of the EMU was supported by a test stand.

\section{F. Test Subject Selection}

Eight subjects were selected, four male and four female, based on their hand length, middle finger length, and hand and middle finger circumferences. This selection was meant to vary the hand size of the subjects, in an attempt to ensure that the developed requirements would be applicable to the full range of potential astronauts. However, since very small and very large subjects cannot be fit adequately in an EMU, future glovebox testing is currently being planned, to check the requirements numbers against the population extremes. Testing was completed with the approval of the JSC Committee for the Protection of Human Subjects, and with appropriate medical monitoring. 


\section{G. Selection of Gloved Conditions}

Testing was initially planned for two gloved conditions and a barehand condition. The unsuited, barehand condition was chosen to allow a baseline against which all other conditions would be compared. The other two planned conditions were an unpressurized ( $0 \mathrm{psi}$ ) case and a pressurized, $4.3 \mathrm{psi}$ condition.

However, interest was expressed in benchmarking the glove's performance without its TMG. In particular, stakeholders were interested in evaluating the manipulation of a vehicle interface device called the Cursor Control Device (CCD) without the TMG. Because the TMG would have to be removed for this evaluation, it was decided to take some additional metrics while the glove was in this condition. Specifically, gloved strength and mobility (including sphere pickup) were also evaluated at 0 and 4.3 psi without the TMG. In the interests of time, tactility and functional EVA tasks were not evaluated without the TMG.

\section{Descriptions of Chosen Tests}

After the list of tests had been finalized, work was done to flesh out the details of each test, and the order and gloved conditions in which they would be completed.

\section{H. Strength}

Three types of strength data were collected: grip strength and two pinch strengths. The pinches tested were a lateral pinch (the so-called key pinch) and pulp pinch. The key pinch involved a similar posture to that seen while gripping a key, while the pulp pinch involved pressing together the pads of the thumb and index finger. These postures are all shown in Figure 5, below.

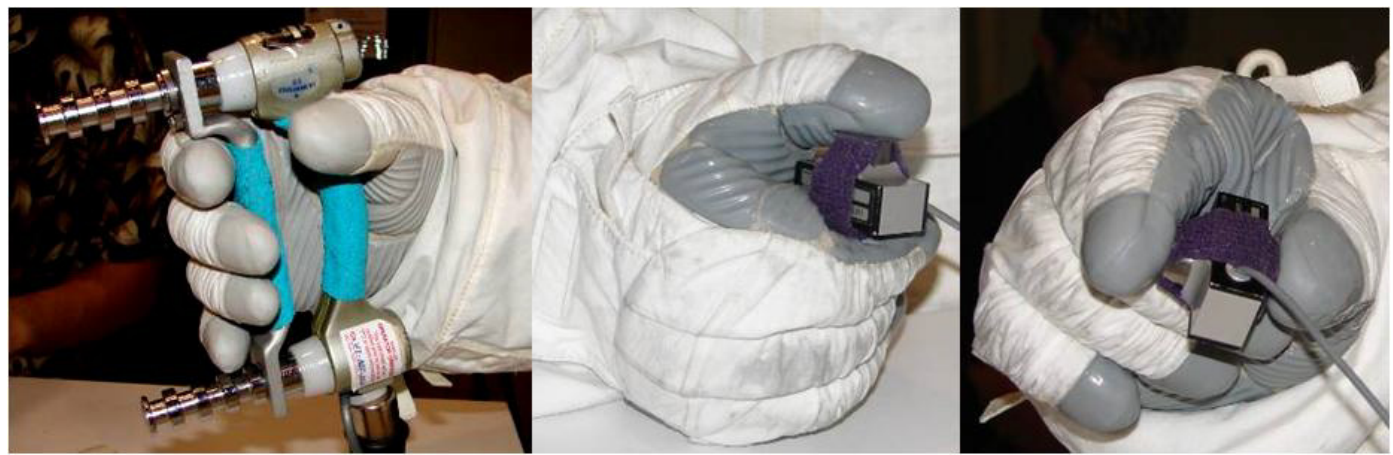

Figure 3. Grip (Left), Key Pinch (Center) and Pulp Pinch (Right) Strength Testing

Grip strength was evaluated using a JAMAR grip dynamometer at an optimal grip span, while pinch strength was measured using a load cell. For each type of testing, the subject was asked to complete a series of three maximal exertions with a break of two minutes between trials to minimize fatigue effects.

\section{Finger Mobility}

The method chosen for quantification of finger mobility was a novel finger sweep method, as inspired by Kuo et al. in their 2008 paper [8]. This evaluation required subjects to drag each finger through a sweep that bounded the full range of motion of that digit. The fingertip sweeps were tracked by attaching a retroreflective marker to the tip of each finger (as seen in Fig. 3), and placing four markers on a rigid plate at the back of the hand. 


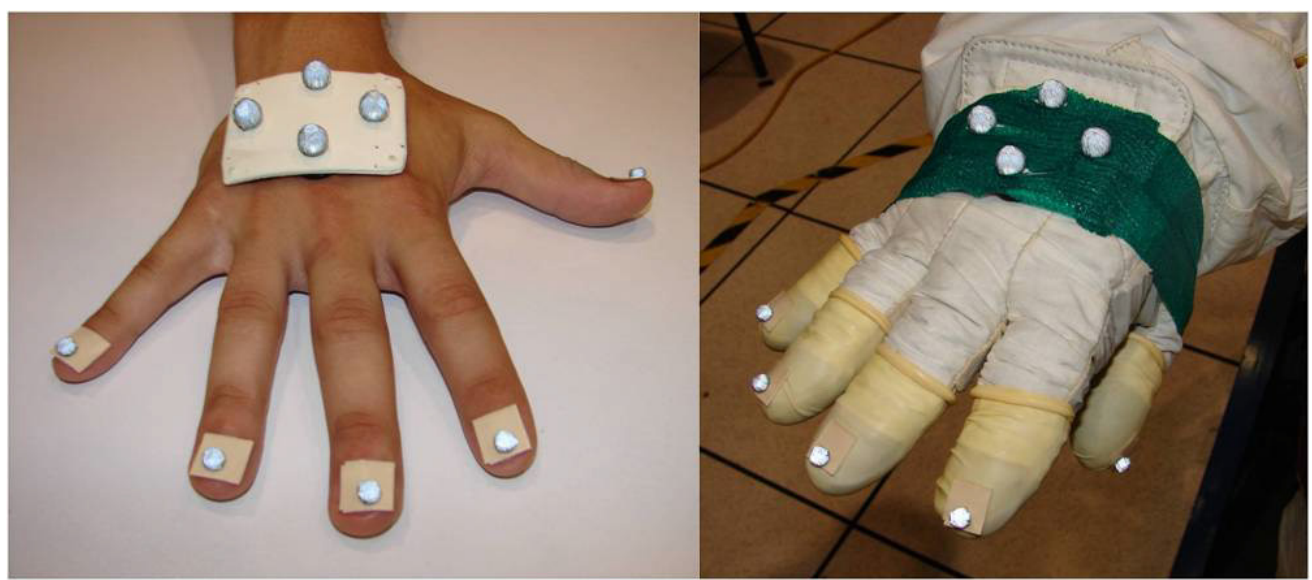

Figure 4. Example of Markers on an Ungloved and Gloved Hand

Finger cots, which are normally used to keep fingertip bandages dry, were placed over the fingers of the Phase VI glove. The markers were then affixed to these, since the double-sided tape did not adhere well to the TMG. The hand plate attached to the back of the hand and/or glove was used to develop a local coordinate system, to define the motion of the fingertips relative to the hand. The motion of the retroreflective markers was tracked in 3D space, by a 12-camera Vicon MX series motion capture system (Oxford Metric, Oxford UK) operating at $100 \mathrm{~Hz}$.

Each subject was asked to complete multiple sweeps for each finger, and the area of the largest sweep was used to define the mobility of that finger. The number of sweeps was initially set at three, but after the first three subjects it was decided to increase the number of sweeps to five to increase the certainty of achieving several clean sweeps.

\section{J. Sphere Manipulation}

Subjects were asked to pick up a $1 / 2$ " diameter marble and a series of Styrofoam spheres ranging from 1" to 8 " in diameter, to evaluate the optimal grip size for the Phase VI gloves. The spheres were covered in fabric tape, in an attempt to reduce the friction between the glove and the sphere. (Less sticky than duct tape, for instance, where the subject could hold on to even the large spheres by just using the stickiness associated with the coated fingertips).

The subject was asked to grip each sphere in turn, lift it away from the table and swing it in an arc, to demonstrate that they had full control over the sphere. If a subject obviously struggled to pick up the sphere, or dropped it during the motion, they were considered to have a tenuous grip only, and this was noted on a data entry sheet. The process was continued until the 8 " sphere had been picked up, or until the subject could not successfully pick up and manipulate a sphere.

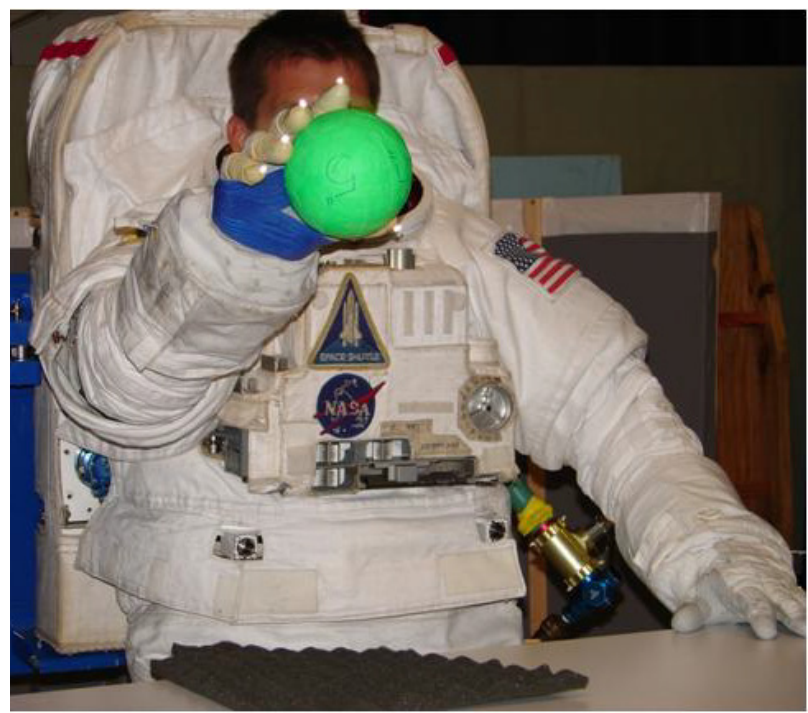

Figure 5. Sphere Manipulation Task

As seen in Figure 4, the Vicon markers were kept on the fingertips during the sphere pickup test, in the interests of potentially using this data in the future to evaluate the posture of the hand while gripping the sphere. Because the finger cots used to attach the markers were naturally slippery, manipulation of the larger sizes of sphere was then repeated without the finger cots, to determine if they had impacted the subject's ability to complete the activity.

\section{K. Tactility}

Tactility of the gloves was evaluated in a novel method that utilized a series of 30 resin blocks with raised bumps. The bumps varied in height and width, and were presented to the subject in a randomized order. The load applied to the block was measured, as the subject was asked to press down with their finger until they could distinguish a bump. After finding the bump, the subject was then asked if the current bump was taller or shorter, 
wider or narrower than the previous bump. In some cases a blank block was provided, to check for false positives the detection of a bump that did not exist.

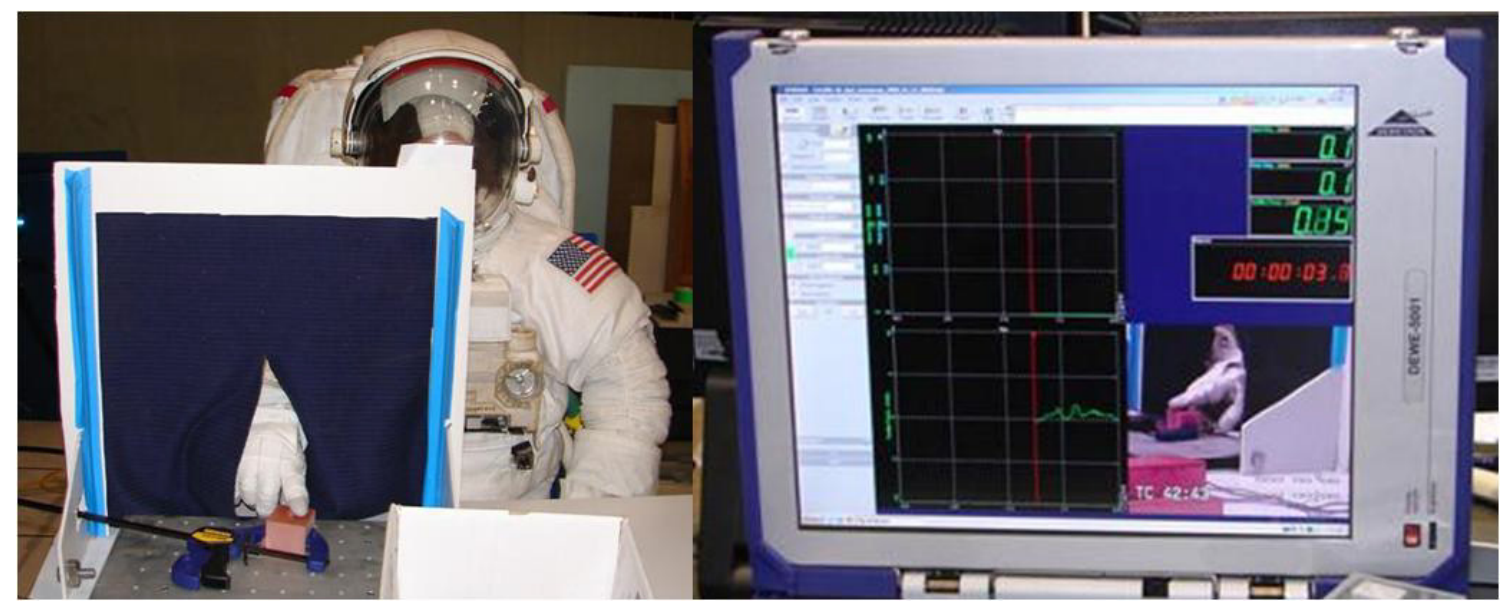

Figure 6. Tactility Testing Setup

As seen in Figure 6, the subject's view was obscured by a curtain, to prevent them from 'cheating' and observing the bump test apparatus. The view on the right shows the data collection setup, where the applied load was displayed real time along with video of the test.

\section{Cursor Control Device Testing}

Task completion time and error data was collected as subjects completed testing with a Cursor Control Device, designed as an interface for the Orion vehicle. The testing involved custom designed software that guided the subject through the test, the physical CCD mockup, and a display that provided feedback to the user.

\section{Functional EVA Tools}

To relate the results of this study back to real-world operations, a series of representative EVA tasks were selected to benchmark the functional performance of the Phase VI gloves. A set of tools was provided by the engineering group, and from these, a series of tasks were developed to challenge the subjects and evaluate the gloves.

The tools included a heavy-duty Pistol Grip Tool (PGT) as seen in, a selection of tethers, a set of wire clippers, a specially designed socket wrench, and a socalled rigidizable tether. All of these tools were optimized to be operated by a suited, gloved crewmember in microgravity, and were good analogs for what an astronaut would actually use on orbit.

The subjects were allowed time to familiarize themselves with each of the tools, and then asked to complete the series of tasks once they felt comfortable handling the equipment. To aid in this familiarization, the subjects were asked to complete an unsuited session with the tools before being asked to manipulate them

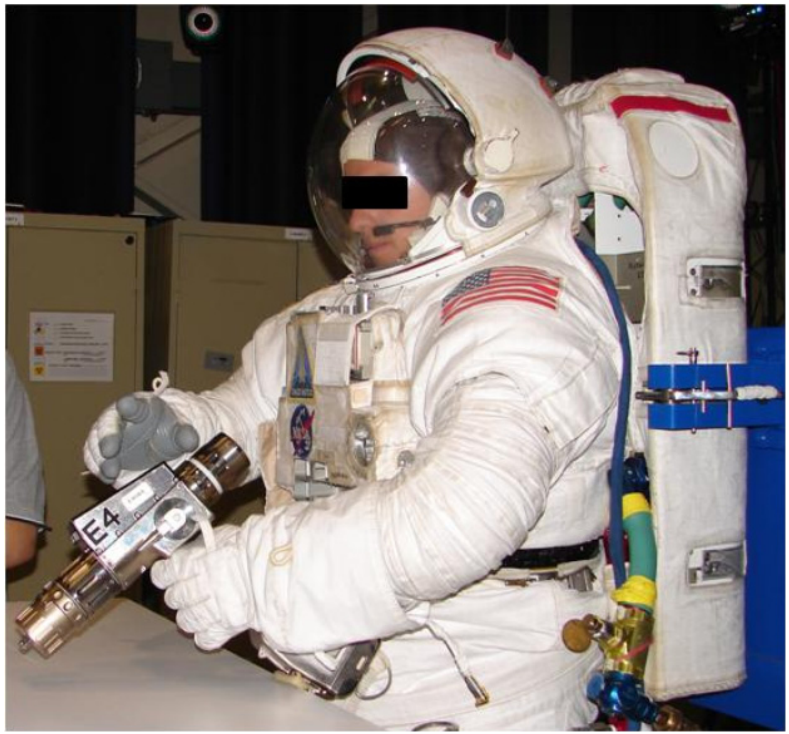

Figure 7. Subject using a PGT while wearing the suit. This was not always possible, however, due to conflicts with tool and subject availability. The tasks were as follows:

1) Complete a series of mode changes (fine and gross manipulation of dials and switches) on a PGT mockup

2) Handle and actuate a set of wire clippers

3) Clip and unclip a tether to the handrail segment and EMU D-Ring 
4) Clip the retractable tether to a handrail section, assemble ratchet assembly and simulate tightening a bolt

5) Attach a rigidizable tether (mounted on the EMU workstation) to the handrail section, and tighten down

One of the most difficult and time consuming tasks ended up being the attachment of a tether to the D-ring on the EMU while suited (see Figure). Issues likely included the reduced tactility while wearing EVA gloves, and the fact that the Dring was out of the subject's field of view (see emphasis, Figure). Several subjects could not successfully complete this task within the time allotted.

The subject's completion time for each task was recorded, and at the end of the functional tools testing they were asked to complete a subjective questionnaire on the difficulty of completing the tasks.

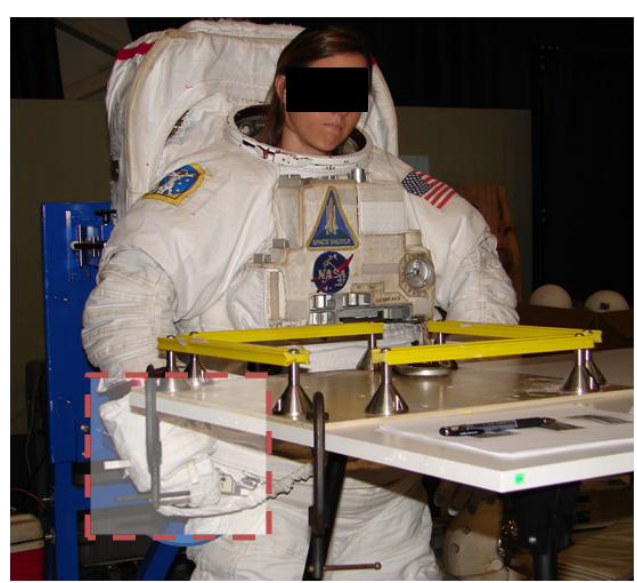

Figure 8. Subject Attempting to Clip a Tether to the EMU D-Ring

\section{Results and Requirement Definition}

Because this paper is meant to focus on the methodology that was used to define gloved requirements, detailed results data will not be included in this document. These results will be presented elsewhere, including papers by Mesloh [9] and Thompson [10] that are currently in press. However, the format of the results is provided, and their application in developing requirements.

\section{A. Strength Data and Requirements Philosophy}

The gloved strength data, recorded in lbf, was examined in terms of percentage of a subject's ungloved strength. The results indicated that for grip strength, the glove's TMG had a noticeable effect on gloved strength - as significant as the decrement associated with pressurizing the glove to 4.3 psi. Pulp pinch strength results were less conclusive, with some strength values jumping in the gloved condition - a phenomenon that was likely a result of either a mechanical advantage in the gloved posture or a subject's ability to 'cheat' and flex their distal knuckle while performing the pulp pinch. For detailed strength data, see [9].

There were many options for developing a strength requirement. For instance, the authors could have used the absolute strength values, or report percentage of unsuited. It was quickly decided that the glove requirements would be best developed using a percentage of ungloved strength, vs. an absolute strength value. This way, when the requirement was applied to a new glove with potentially different test subjects, their baseline strength would not have an effect on the glove meeting or failing the requirement. A subject would only need to achieve the same percentage of their unsuited strength that this study's subjects had been able to achieve wearing a Phase VI glove.

Another decision involved the use of mean, max or min strength values to develop requirements, or some combination of the same. If the requirements were developed using the minimum strength values, or the largest strength decrement seen while wearing Phase VI gloves, than the new glove design would be expected perform, on the average, worse than the current glove. If mean values were used, the glove would be required to perform similarly to the Phase VI. If max strength values (minimum strength decrements) were used,

\section{B. Mobility Requirements}

The mobility data was processed by finding the area of the largest fingertip sweep for each finger under each of the five testing conditions (ungloved, and 0 and 4.3 psi for the Phase VI glove with and without the TMG). Figure 5 shows a visualization of a finger sweep, and the mesh that is constructed to find the area. 


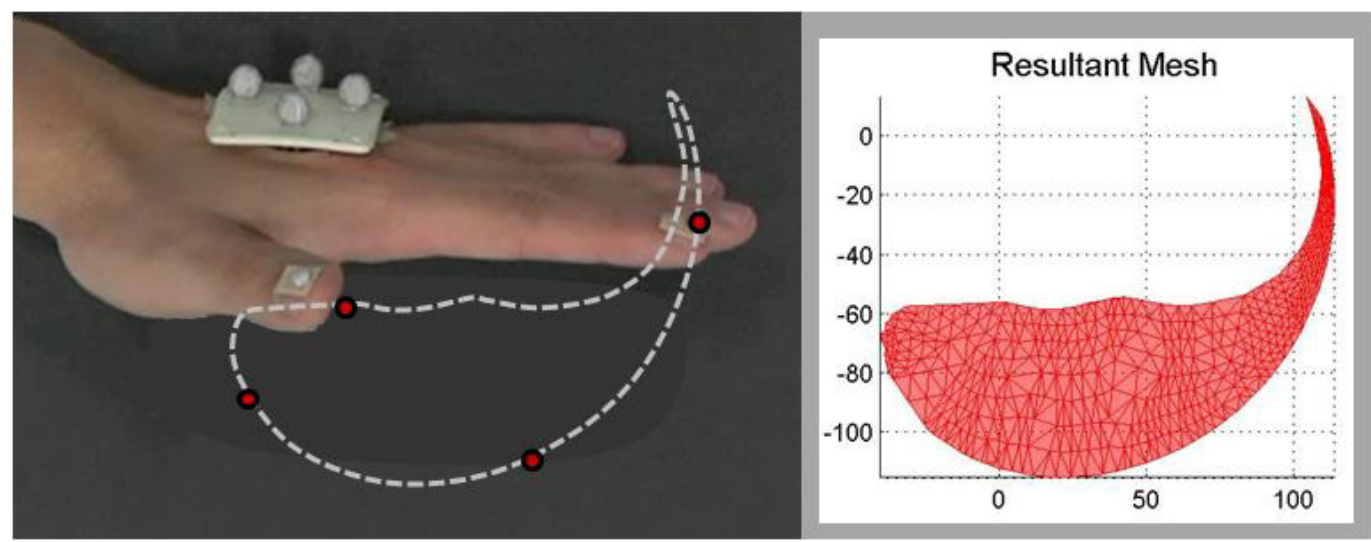

Figure 9. Visualization of an Unsuited Index Finger Sweep, and Associated Area Mesh

For each subject, sweep areas were calculated for each finger, for all gloved conditions. Each subject's suited finger mobility was always related back to the unsuited mobility of that subject for the given finger. For example, a subject might complete an index finger sweep unsuited and see a sweep area of $5000 \mathrm{~mm}^{2}$, but could perform the same motion wearing a glove and form a much smaller sweep. This index finger sweep area was reduced by as much as $80 \%$ for a subject wearing a pressurized glove with a TMG integrated.

The mobility testing resulted in a table of values for fingertip sweep areas. These areas were reported as a fraction of the subject's unsuited finger sweep area, and then compared across subjects and gloved conditions. This normalization was completed to minimize the effect of finger length on the results, since subjects of varying hand size were used in the evaluation.

\section{Tactility Requirements}

Tactility data was provided in terms of the force required to detect a bump, and the amount by which this exceeded the unsuited requirements for bump detection. Additional data included incidence of false positives (detecting a bump where one did not exist) and false negatives (not finding a bump when one existed). Subjects were also asked if they could successfully determine whether a bump was shorter, taller, narrower or wider than the previous bump. Tactility maps were also provided that showed the 'sweet spot' of bump height and width that was most easily detected, especially by a subject wearing a pressurized EVA glove.

Gloved tactility requirements were provided in terms of the threshold index fingertip tactile-force at which a bump of a given height and width had to be detected. This data could also be employed by spacecraft designers, while selecting a size of switch or button that needed to be detected by a gloved hand. The threshold detection force could also be used to ensure that a gloved crewmember would not accidentally bump such a button, while attempting to search for switches with their finger. For detailed tactility data, see [10].

\section{Cursor Control Device Data}

Results from the Cursor Control Device testing fed into design recommendations for vehicle interface devices, and were not used directly to develop the glove requirements. However, the time of completion for the CCD portion of testing was provided for unsuited, gloved unpressurized and gloved pressurized cases, without the TMG integrated. Testing was completed without the TMG to allow a better analog to the gloves that crewmembers would wear inside the vehicle, where they would interact with the device.

\section{E. Functional EVA Tools Data}

Time to completion was recorded for each of the tasks performed using EVA tools. The testing conditions were ungloved, gloved unpressurized and gloved pressurized. In the interests of time, this testing was not completed with TMG removed. Results from the EVA tool testing did not feed directly into requirements, but were provided as reference. Trends in the data were as expected, with performance time generally increasing from ungloved to gloved, and again with glove pressurization. The task with the greatest delta from ungloved to gloved was the tether unclipping task, where the subject was asked to unfasten and remove a tether that had been previously attached to the EMU's D-ring, out of their field of view. 


\section{Conclusion}

The results from this study fed directly into requirements for a next generation glove, and could also be utilized for the design of spacecraft controls or EVA tools that the glove is meant to interact with. This same methodology could also be applied to the evaluation of other protective gloves and to aid in design refinements by benchmarking prototypes at all stages of development.

Future planned glove testing includes a glovebox test, meant to include a larger range of subject sizes and also a series of intermediate pressures in addition to the 0 psi and 4.3 psi included in the current Phase VI glove testing. Testing of very large and very small people was not possible in the suited glove testing due to the requirements of the EMU, which can only be sized for a limited range of subject anthropometry. Another area of research will be gloved testing at pressures higher than the Phase VI's 4.3 psi operating pressure. This type of testing will be critical, since some off-nominal operational concepts require a suit to operate at pressures as high as 8 psi. Little data is available within this operating range, because testing at such a high pressure is considered destructive of the suit hardware. A separate proposed glove box test would investigate the glove's performance at higher operating pressures, but would likely involve a smaller number of tests due to constraints associated with the specially designed high pressure glovebox.

\section{Acknowledgments}

The author wishes to thanks Greg Moroney of the Crew and Thermal Systems Branch for his help and support, and Angela Lesser and her associates from the suit group, as well as the Constellation EVA Project Office for funding this endeavor. They would also like to credit Giaccari Luigi, whose open source meshing software was utilized to aid in meshing the 3D finger sweeps.

\section{References}

\footnotetext{
${ }^{1}$ Vatistas, G. H., Lin, S., and Kwok, C. K., "Reverse Flow Radius in Vortex Chambers," AIAA Journal, Vol. 24, No. 11, 1986, pp. 1872, 1873 .

${ }^{2}$ Dornheim, M. A., "Planetary Flight Surge Faces Budget Realities," Aviation Week and Space Technology, Vol. 145, No. 24, 9 Dec. 1996, pp. 44-46.

${ }^{3}$ Terster, W., "NASA Considers Switch to Delta 2," Space News, Vol. 8, No. 2, 13-19 Jan. 1997, pp., 1, 18.

All of the preceding information is required. The journal issue number ("No. 11" in Ref. 1) is preferred, but the month (Nov.) can be substituted if the issue number is not available. Use the complete date for daily and weekly publications. Transactions follow the same style as other journals; if punctuation is necessary, use a colon to separate the transactions title from the journal title.
} 